\title{
Synthesis of a seco iso-secologanin aglycone analogue of interest towards seco-iridoids and monoterpene indole alkaloids
}

\author{
Hussein Abou-Hamdan, Régis Guillot, Cyrille Kouklovsky, Guillaume Vincent* \\ Institut de Chimie Moléculaire et des Matériaux d'Orsay (ICMMO) \\ Université Paris-Saclay, CNRS, 91405, Orsay, France \\ *guillaume.vincent@universite-paris-saclay.fr
}

\section{Table of contents}

1. NMR spectra of compounds

3. References 
1. NMR spectra of compounds

1H NMR $\left(\mathrm{CDCl}_{3}, 300 \mathrm{MHz}\right)$




$13 \mathrm{C}\left\{{ }^{1} \mathrm{H}\right\} \operatorname{NMR}\left(\mathrm{CDCl}_{3}, 91 \mathrm{MHz}\right)$

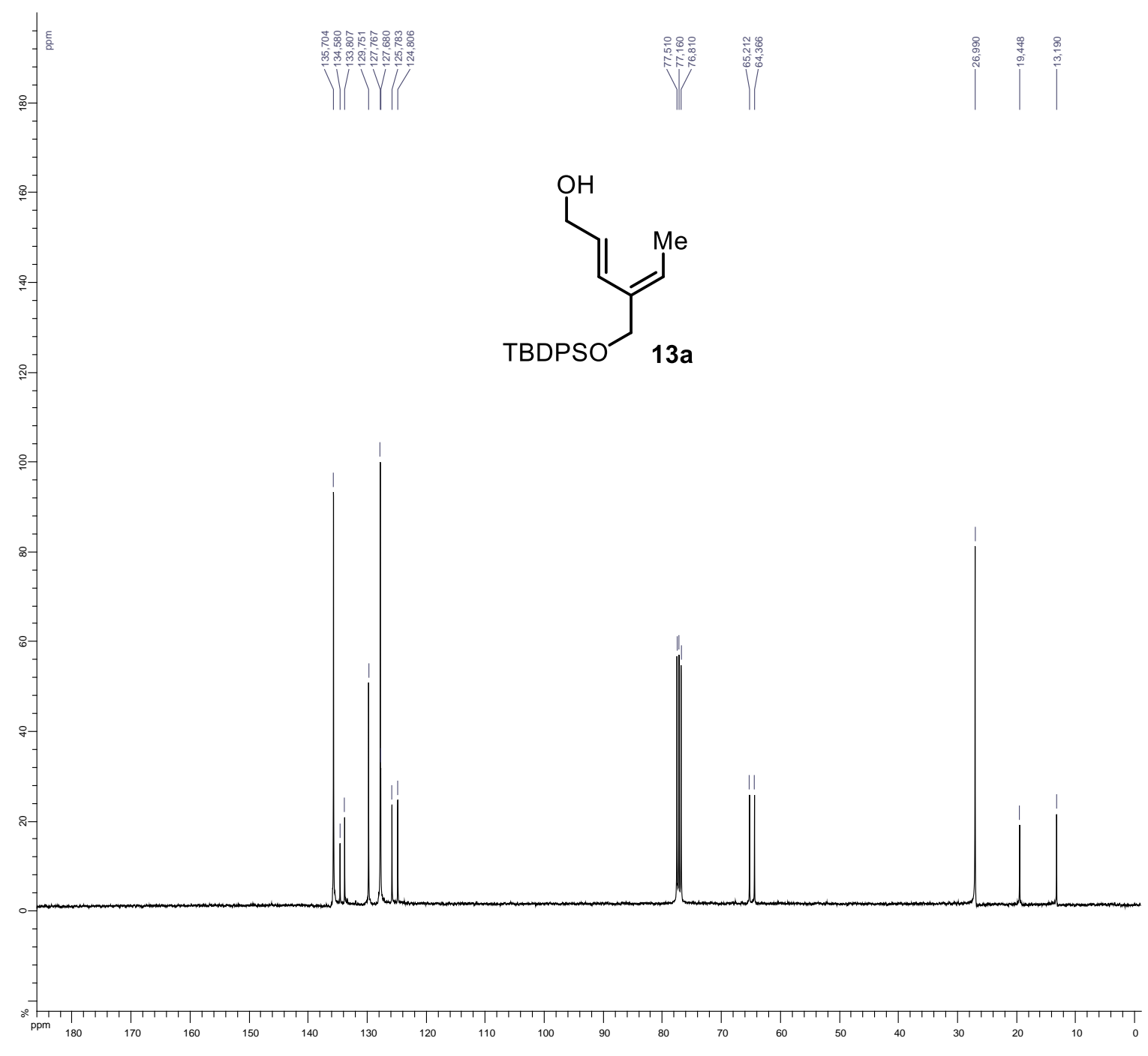


1H NMR $\left(\mathrm{CDCl}_{3}, 300 \mathrm{MHz}\right)$




$13 \mathrm{C}\left\{{ }^{1} \mathrm{H}\right\} \mathrm{NMR}\left(\mathrm{CDCl}_{3}, 91 \mathrm{MHz}\right)$

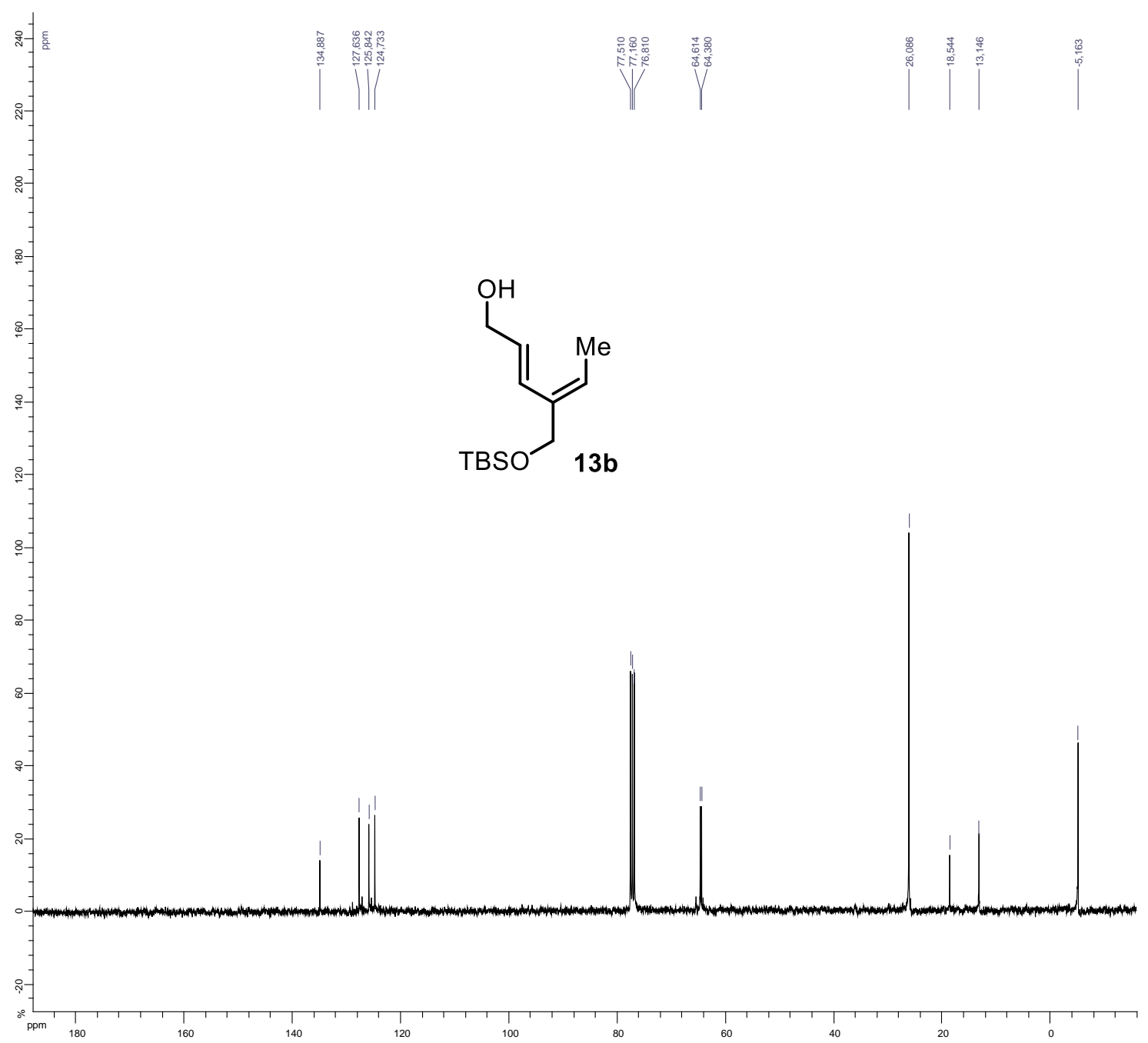


1H NMR $\left(\mathrm{CDCl}_{3}, 360 \mathrm{MHz}\right)$

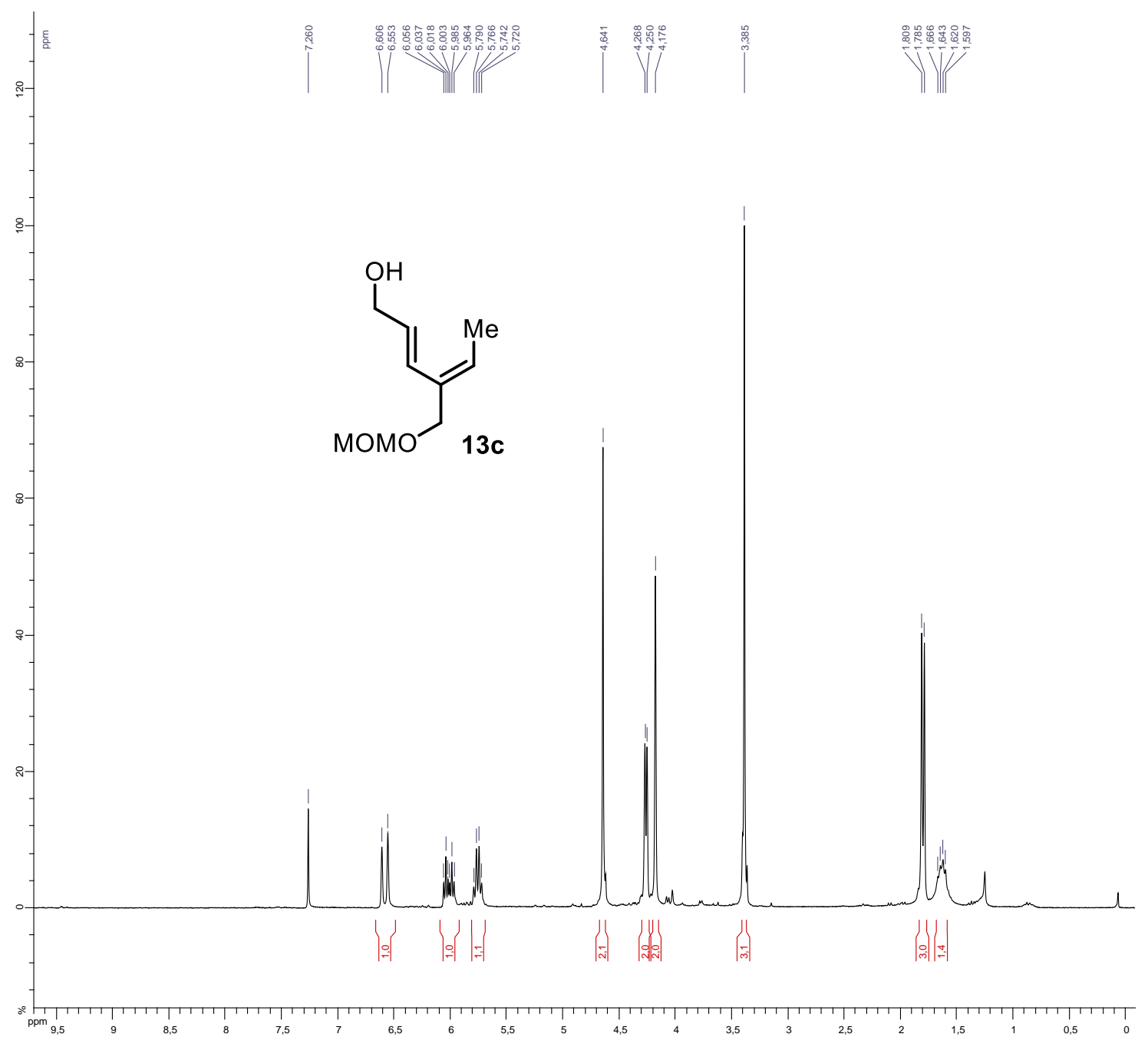


$13 \mathrm{C}\left\{{ }^{1} \mathrm{H}\right\} \mathrm{NMR}\left(\mathrm{CDCl}_{3}, 91 \mathrm{MHz}\right)$

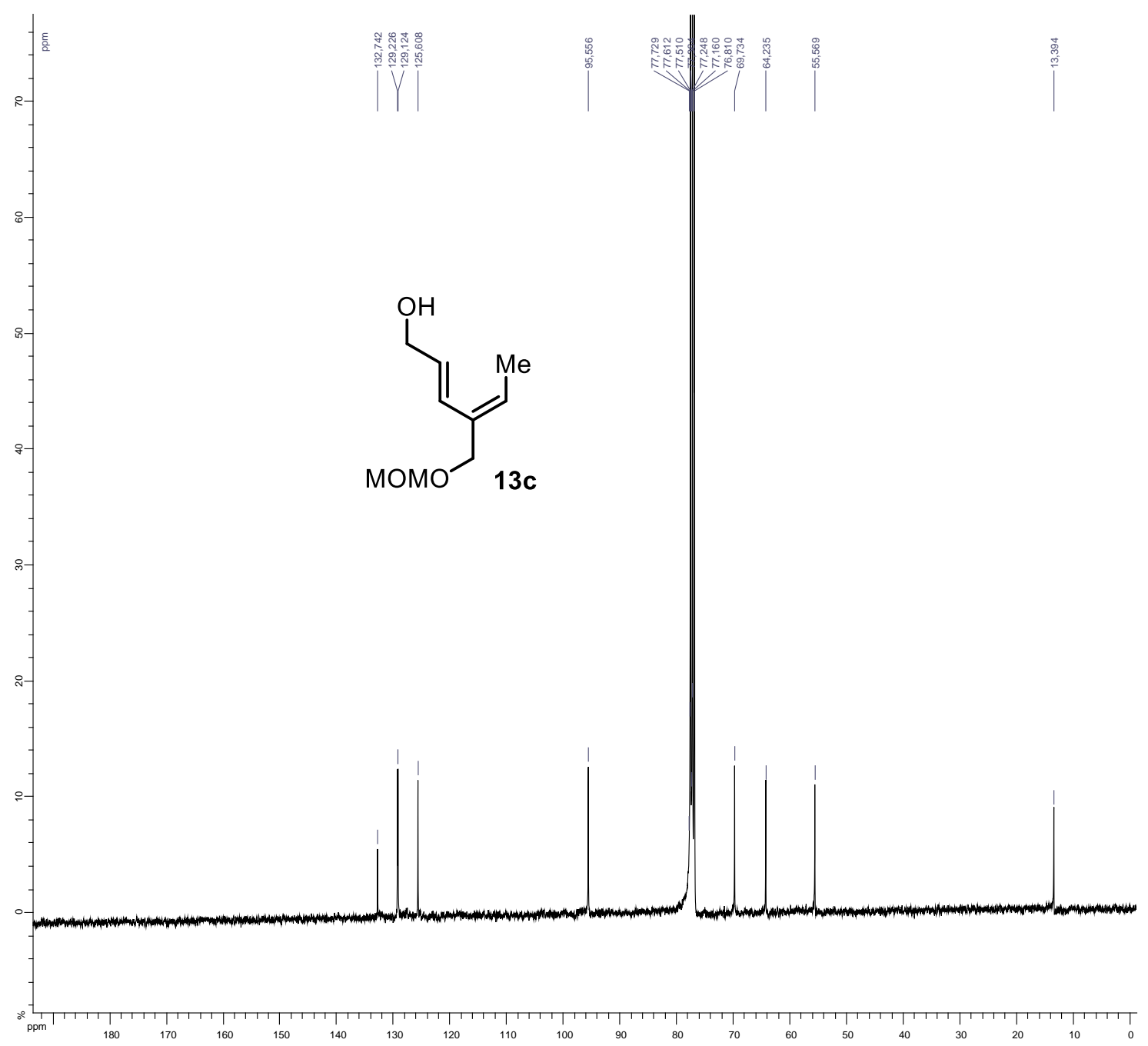


1H NMR $\left(\mathrm{CDCl}_{3}, 360 \mathrm{MHz}\right)$

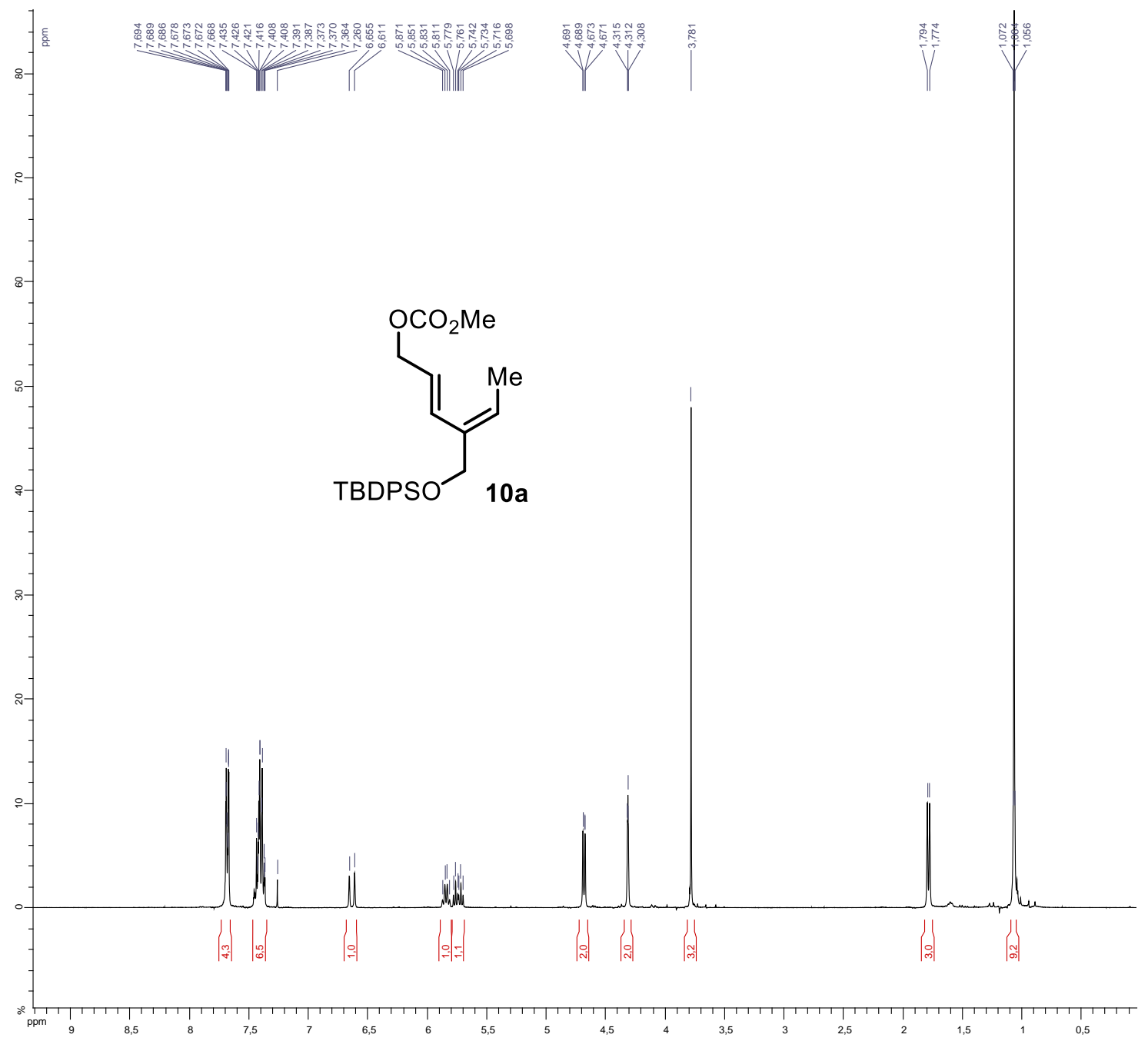


$13 \mathrm{C}\left\{{ }^{1} \mathrm{H}\right\} \mathrm{NMR}\left(\mathrm{CDCl}_{3}, 91 \mathrm{MHz}\right)$

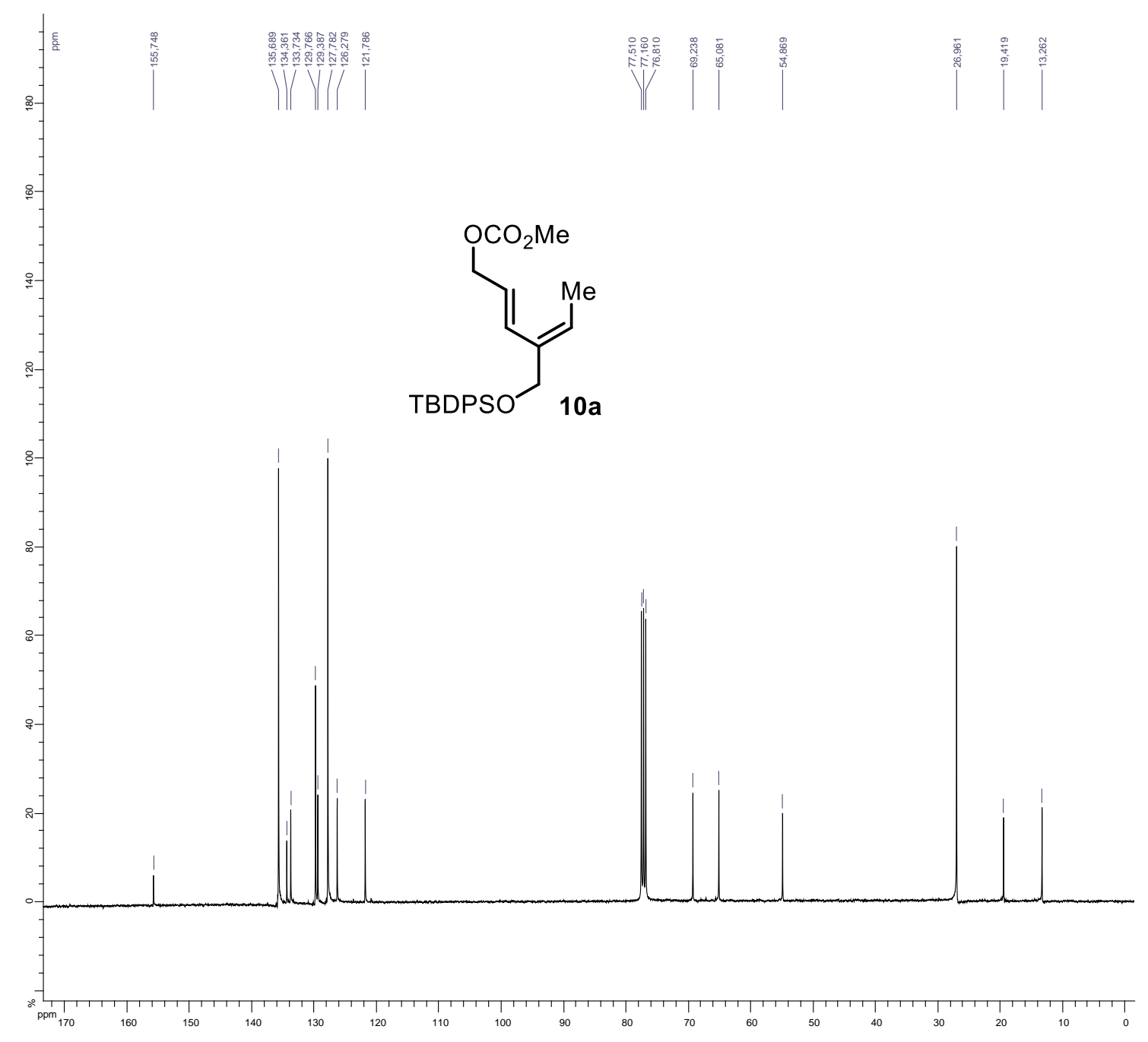


1H NMR $\left(\mathrm{CDCl}_{3}, 300 \mathrm{MHz}\right)$




$13 \mathrm{C}\left\{{ }^{1} \mathrm{H}\right\} \mathrm{NMR}\left(\mathrm{CDCl}_{3}, 91 \mathrm{MHz}\right)$

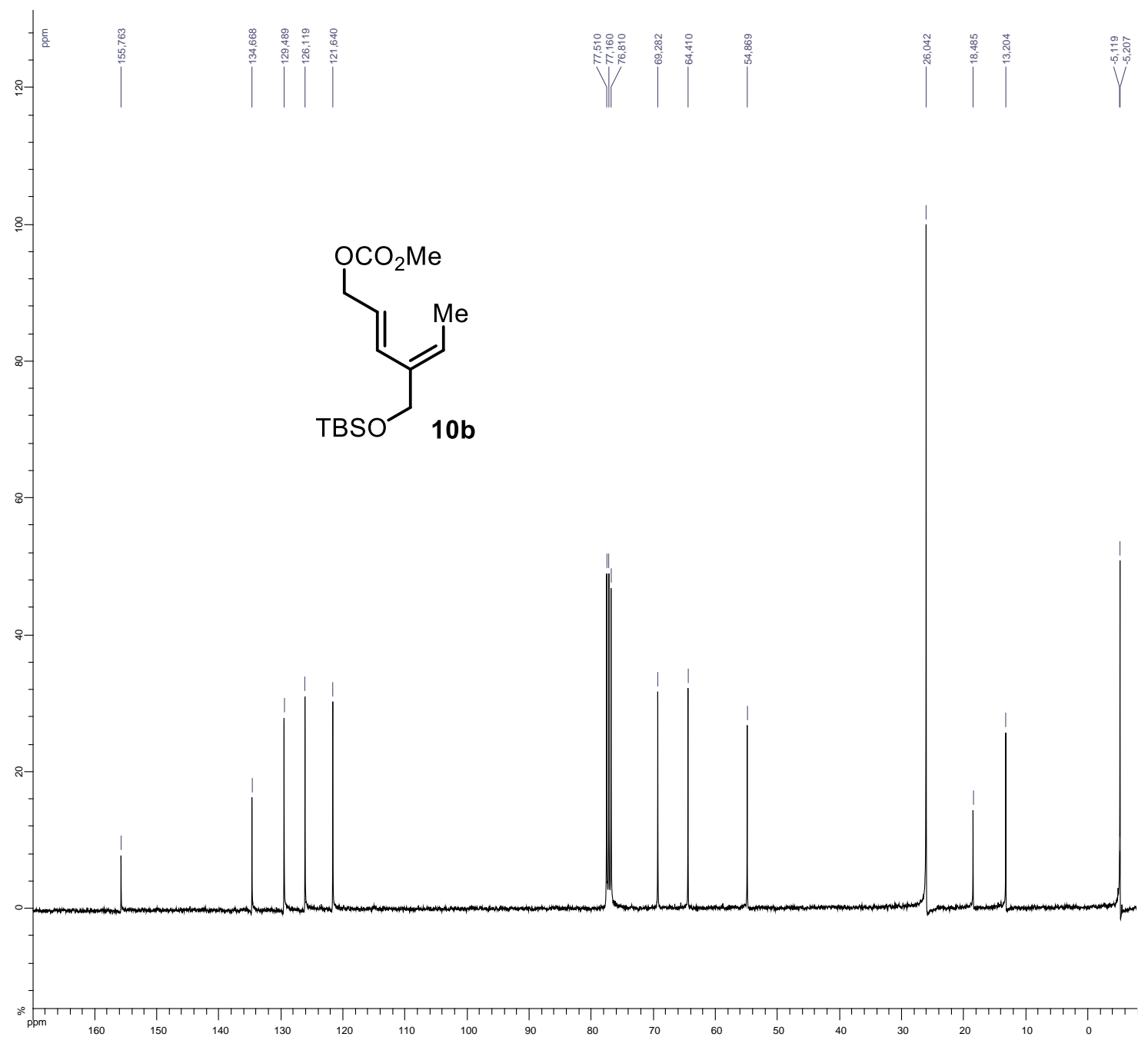


1H NMR $\left(\mathrm{CDCl}_{3}, 360 \mathrm{MHz}\right)$

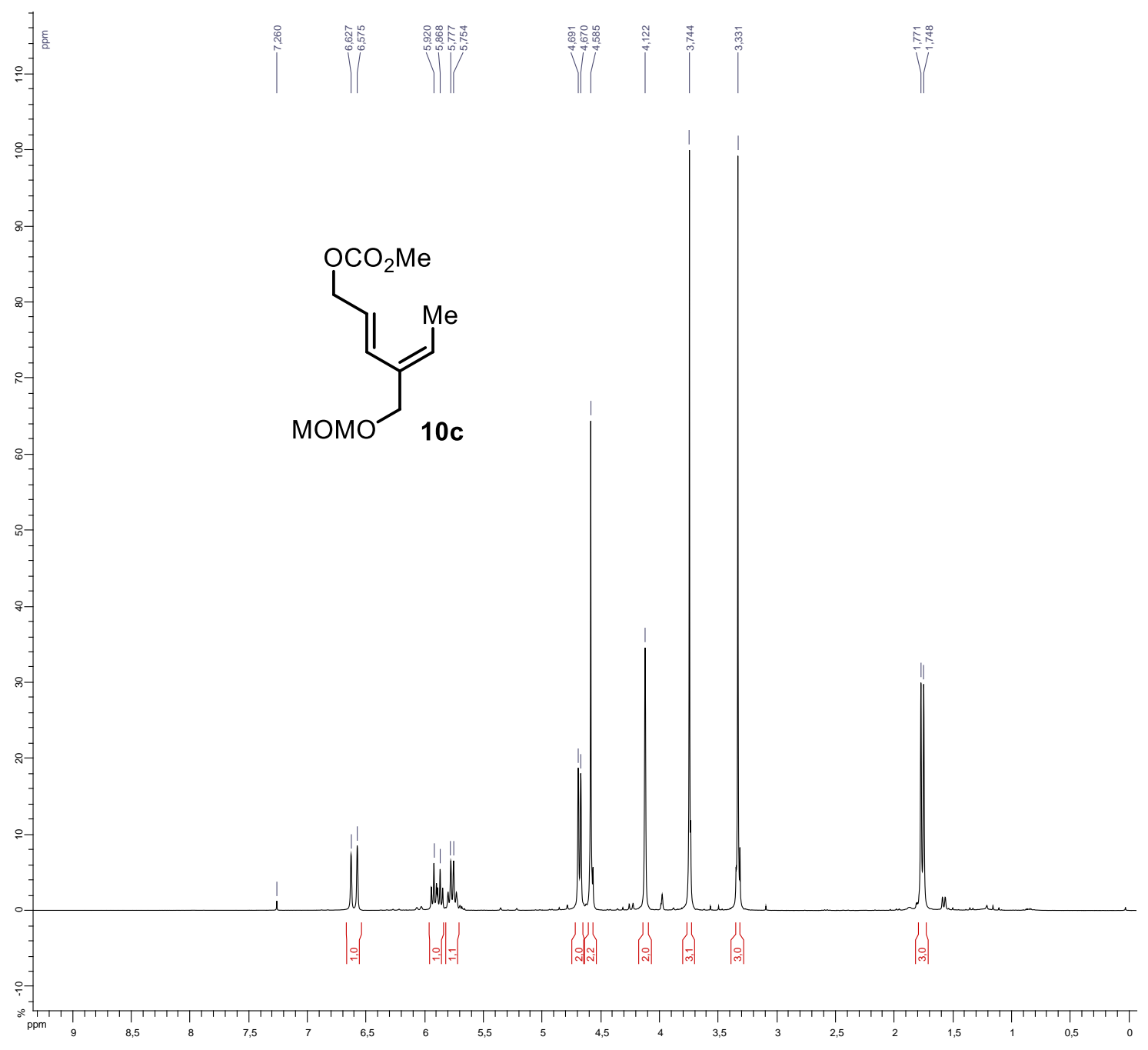


$13 \mathrm{C}\left\{{ }^{1} \mathrm{H}\right\} \mathrm{NMR}\left(\mathrm{CDCl}_{3}, 91 \mathrm{MHz}\right)$

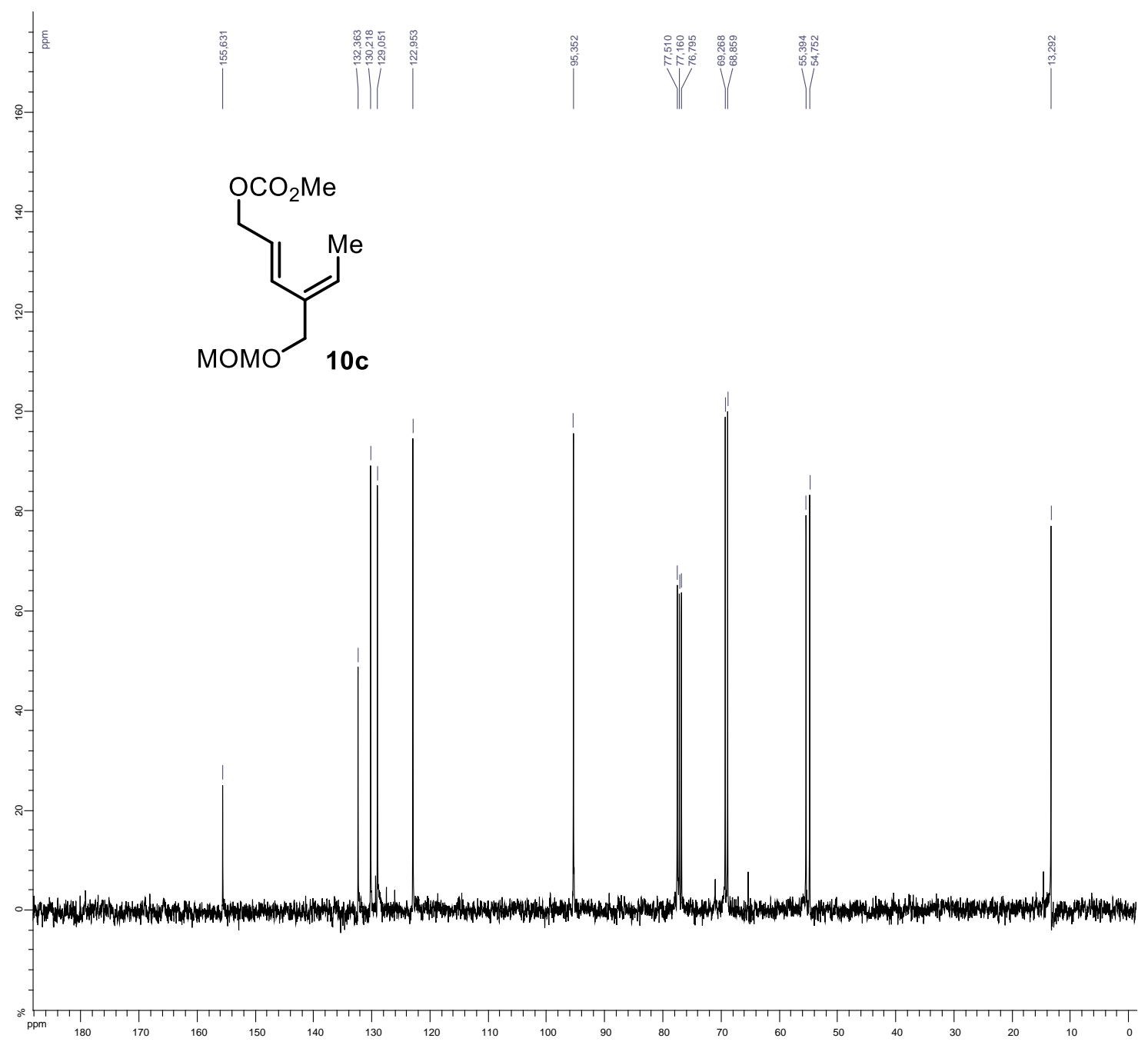


$1 \mathrm{H} \mathrm{NMR}\left(\mathrm{CDCl}_{3}, 300 \mathrm{MHz}\right)$

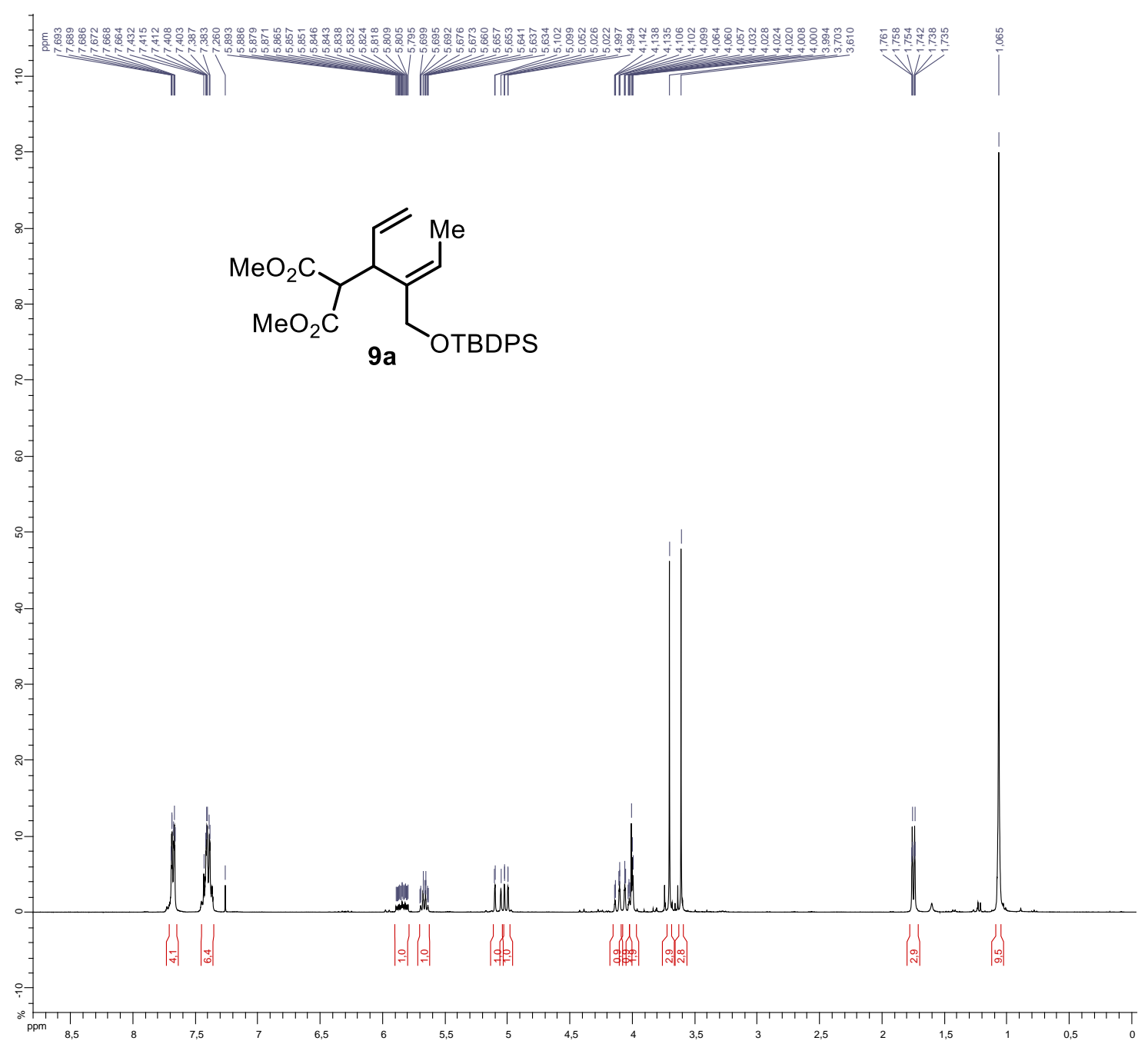


$13 \mathrm{C}\left\{{ }^{1} \mathrm{H}\right\} \mathrm{NMR}\left(\mathrm{CDCl}_{3}, 91 \mathrm{MHz}\right)$

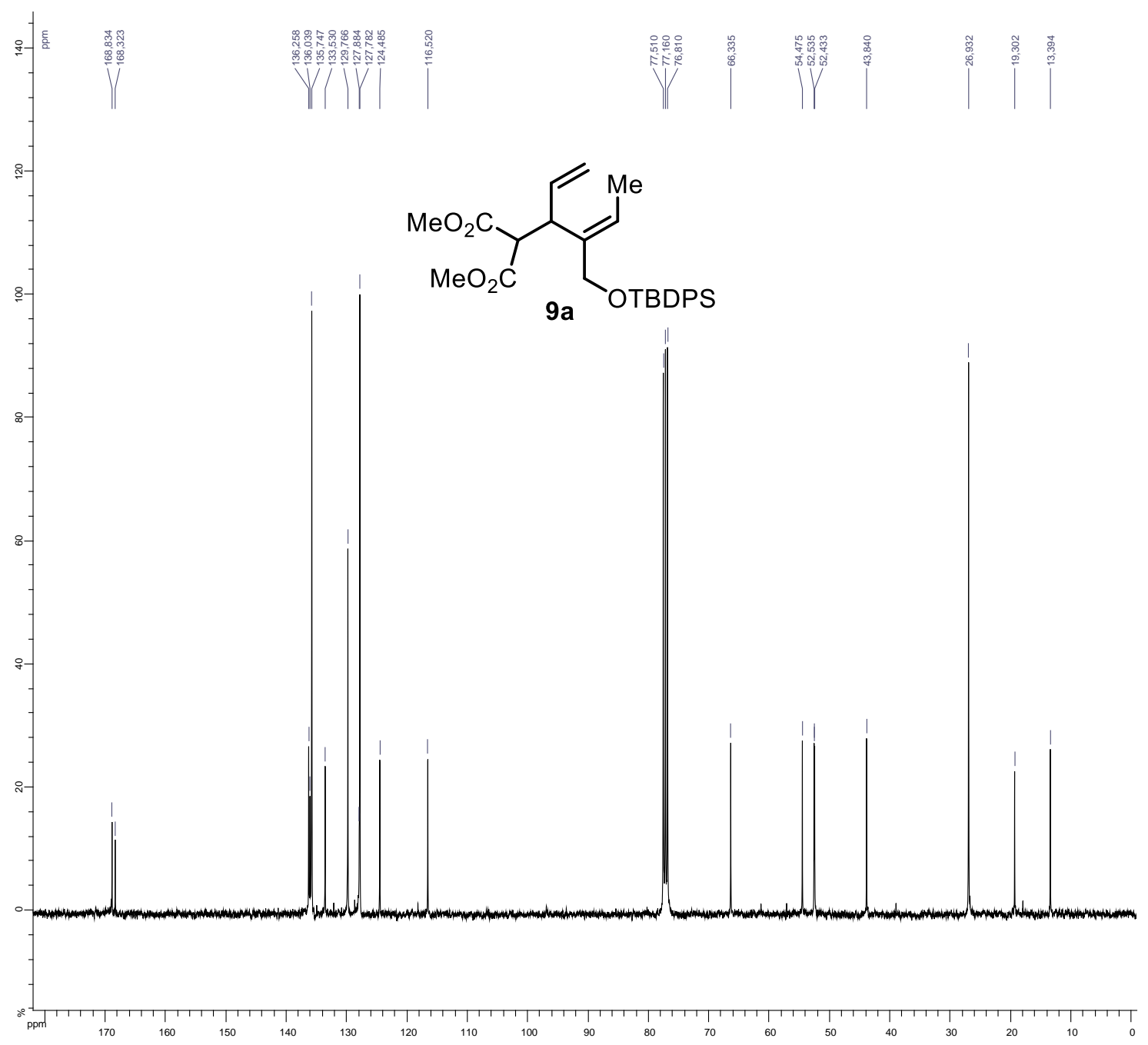


1H NMR $\left(\mathrm{CDCl}_{3}, 360 \mathrm{MHz}\right)$

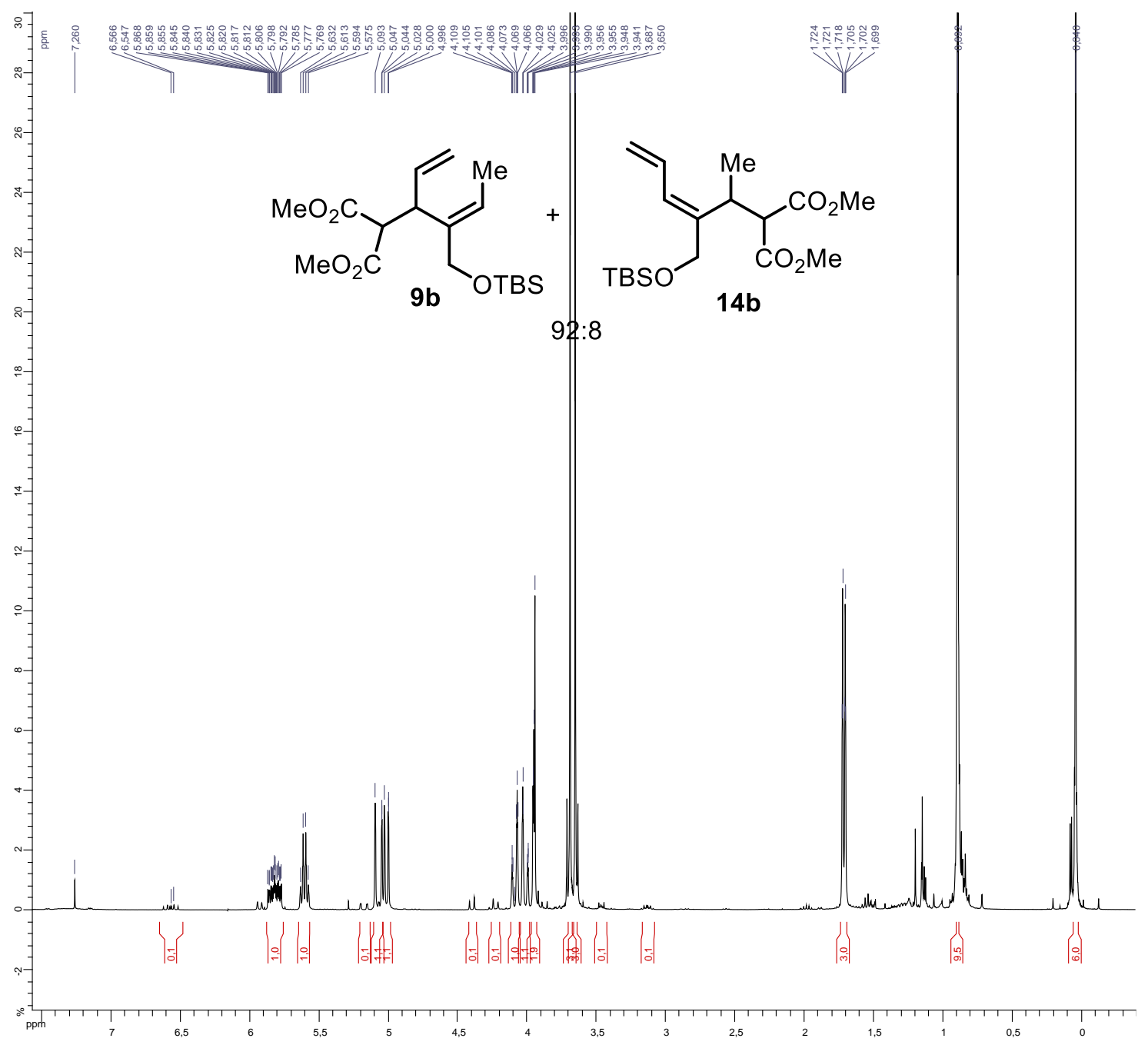


$13 \mathrm{C}\left\{{ }^{1} \mathrm{H}\right\} \mathrm{NMR}\left(\mathrm{CDCl}_{3}, 91 \mathrm{MHz}\right)$

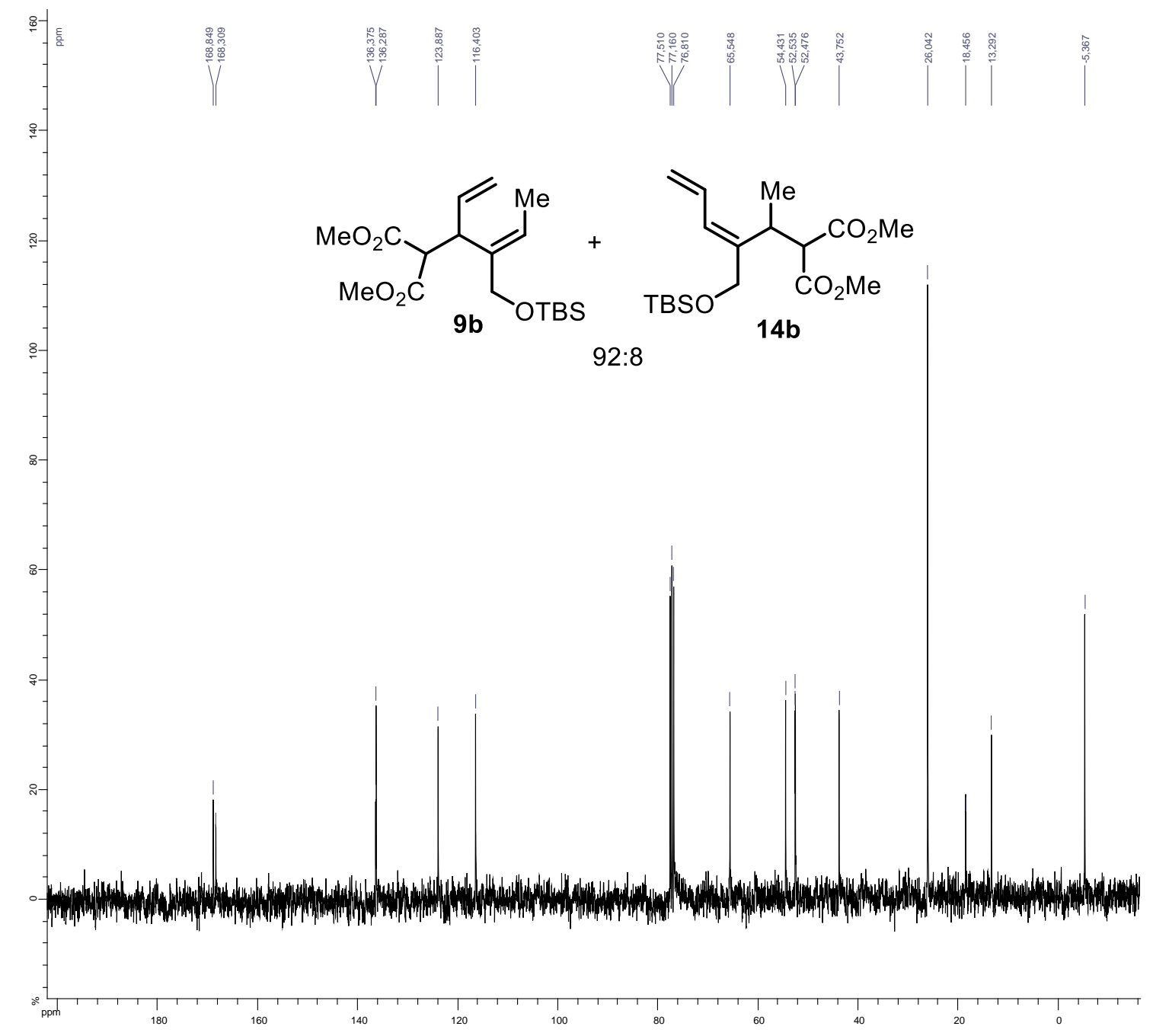


1H NMR $\left(\mathrm{CDCl}_{3}, 300 \mathrm{MHz}\right)$

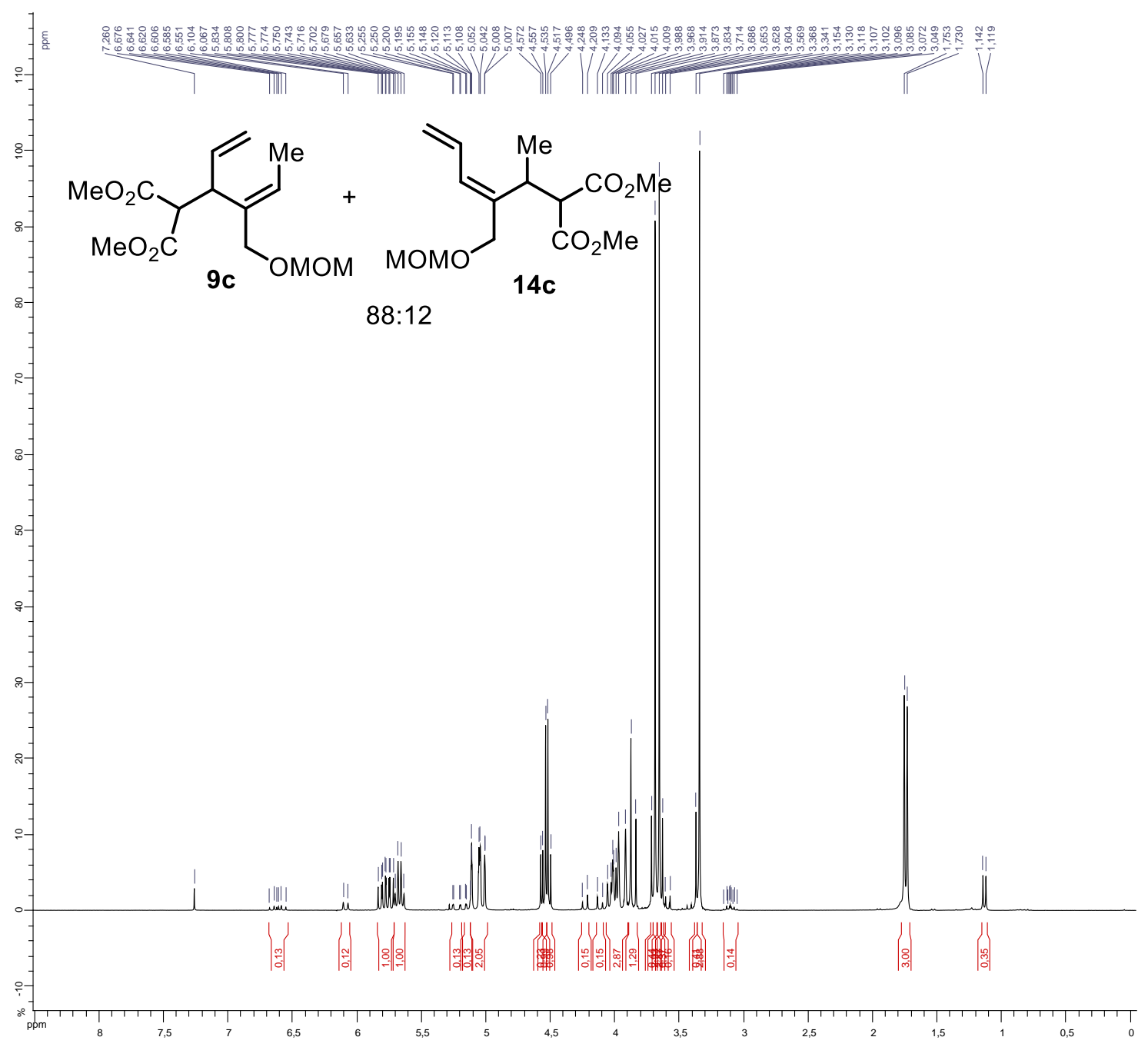


$13 \mathrm{C}\left\{{ }^{1} \mathrm{H}\right\} \mathrm{NMR}\left(\mathrm{CDCl}_{3}, 75 \mathrm{MHz}\right)$

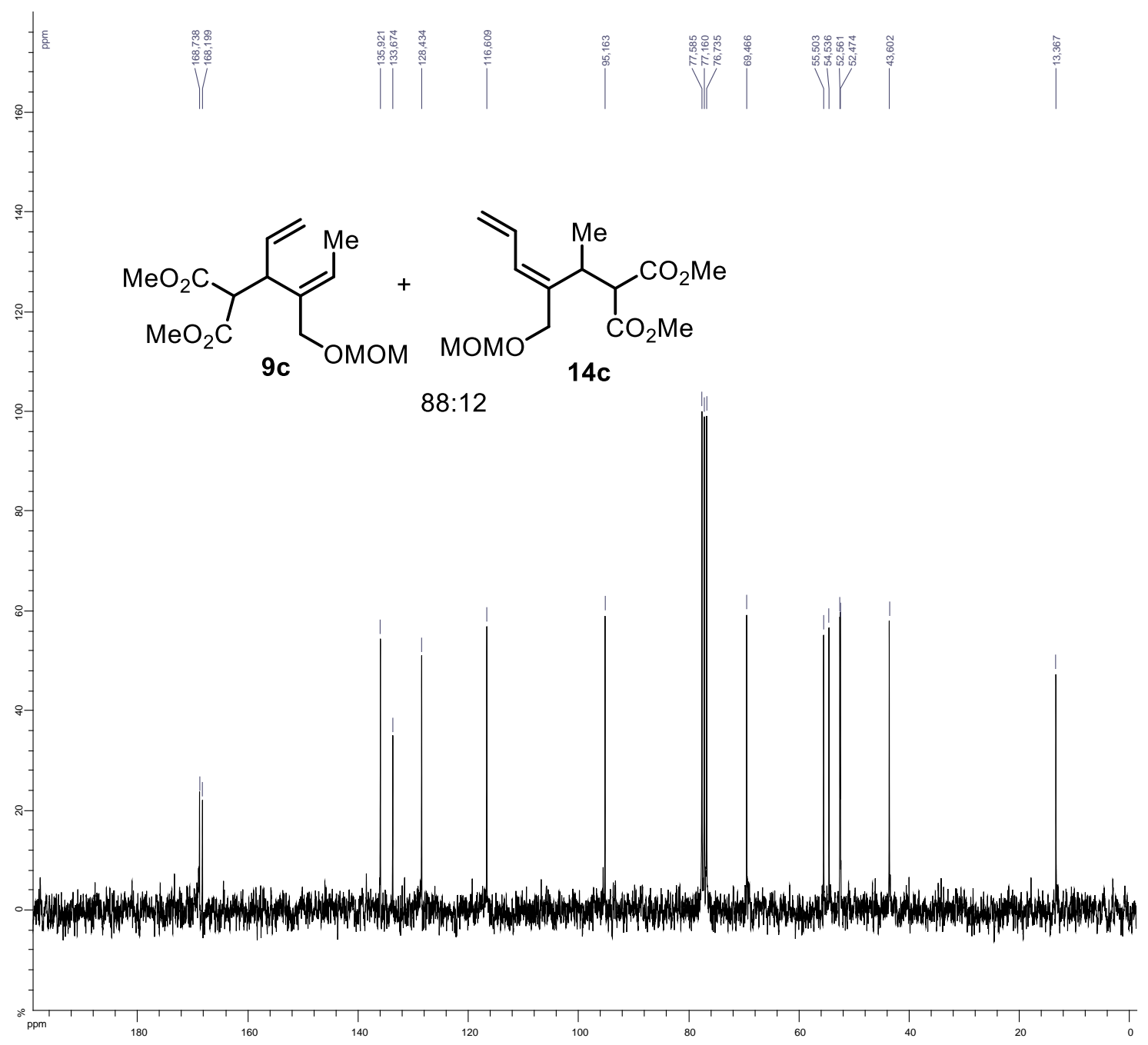


1H NMR $\left(\mathrm{CDCl}_{3}, 360 \mathrm{MHz}\right)$

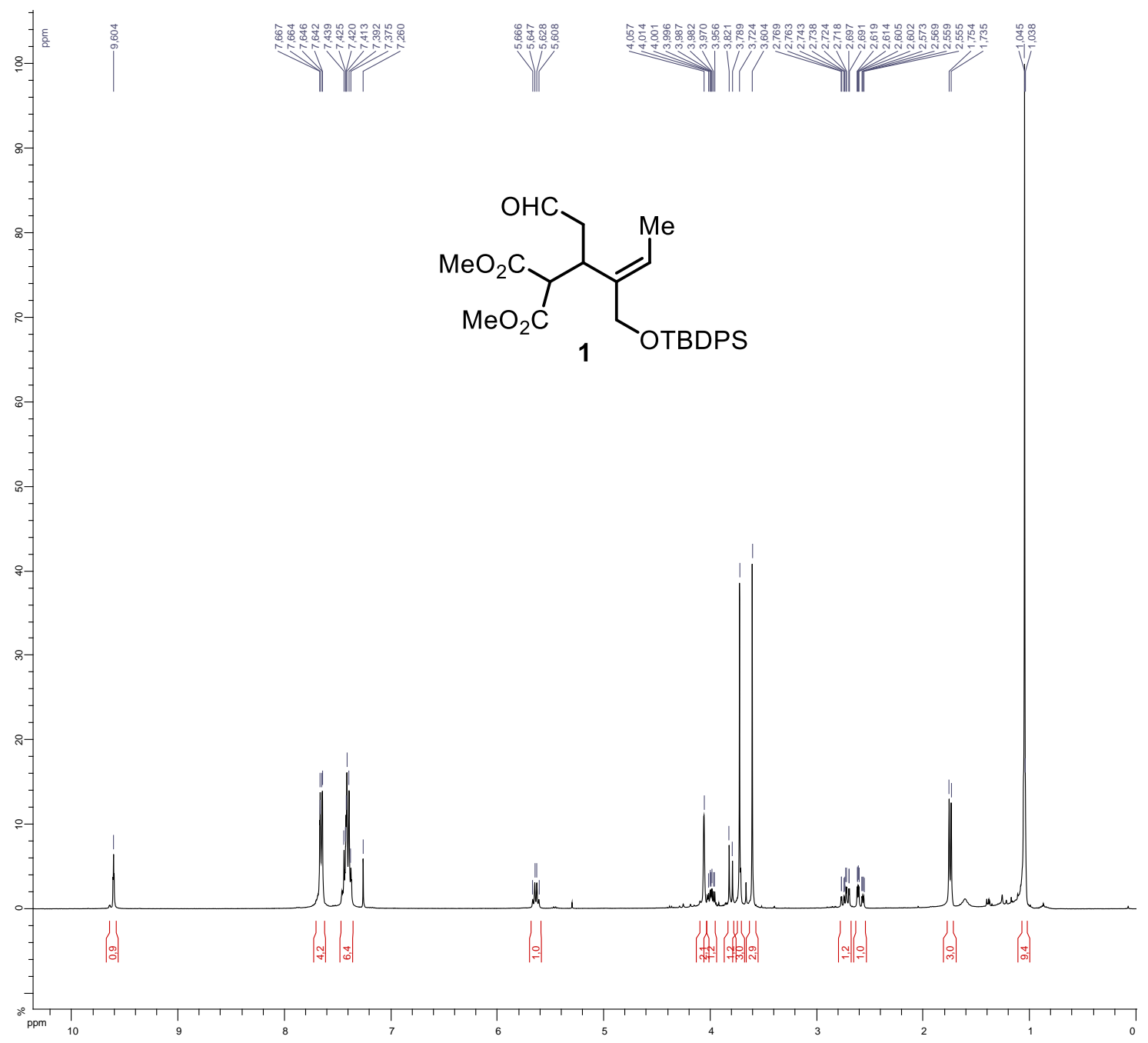


$13 \mathrm{C}\left\{{ }^{1} \mathrm{H}\right\} \mathrm{NMR}\left(\mathrm{CDCl}_{3}, 91 \mathrm{MHz}\right)$

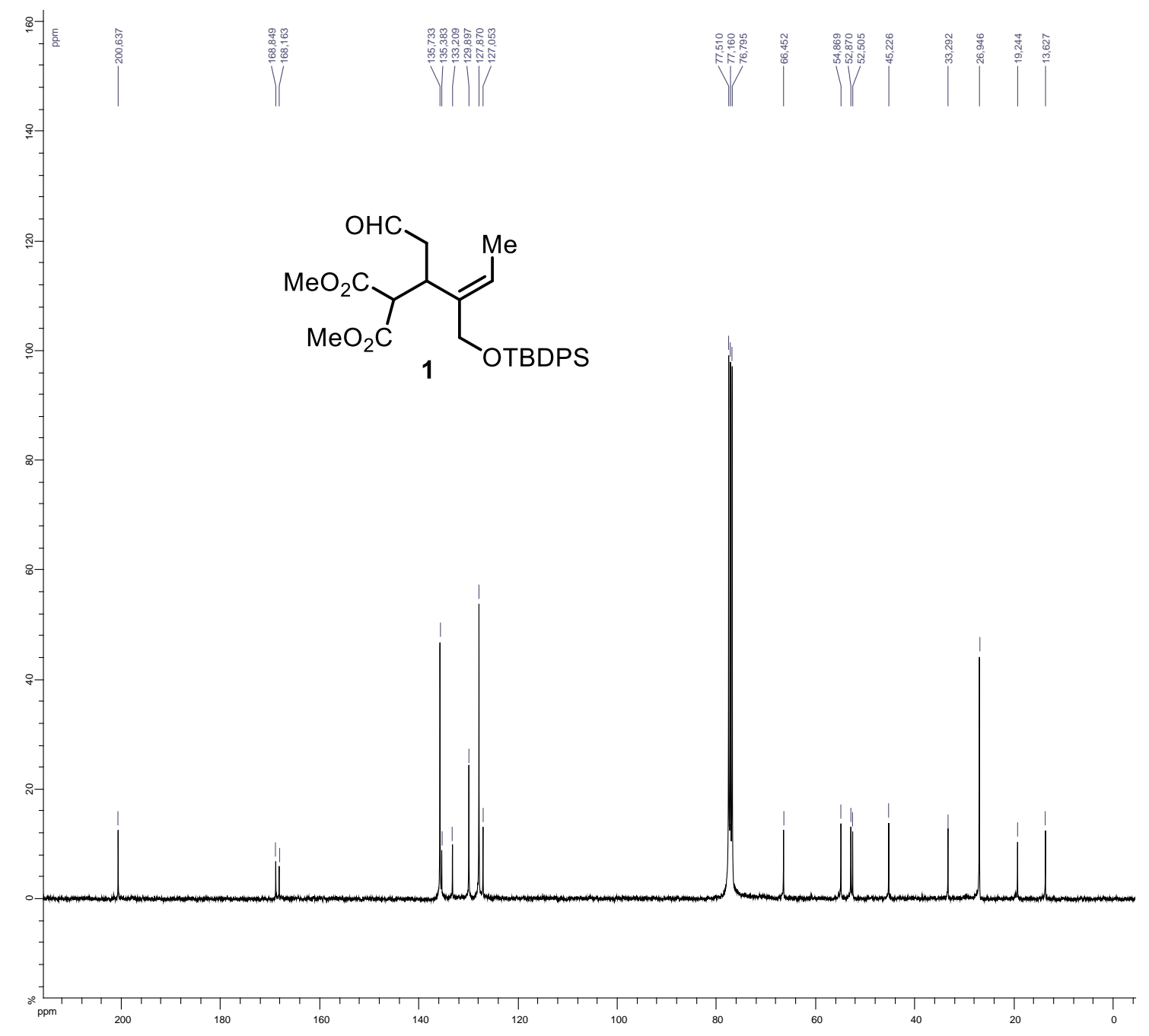


$1 \mathrm{H} \mathrm{NMR}\left(\mathrm{CDCl}_{3}, 300 \mathrm{MHz}\right)$




$13 \mathrm{C}\left\{{ }^{1} \mathrm{H}\right\} \mathrm{NMR}\left(\mathrm{CDCl}_{3}, 75 \mathrm{MHz}\right)$

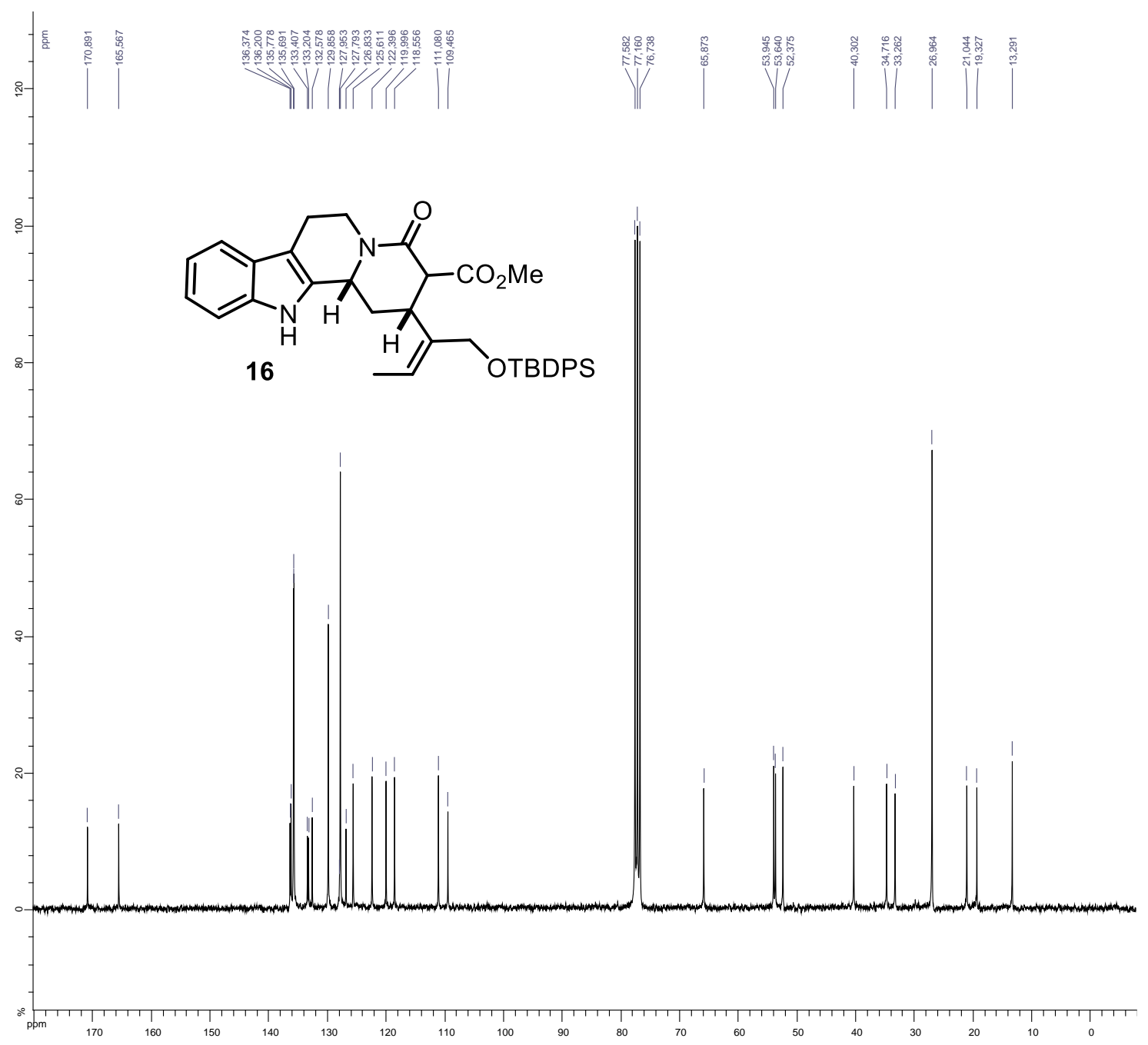


1H NMR $\left(\mathrm{CDCl}_{3}, 300 \mathrm{MHz}\right)$

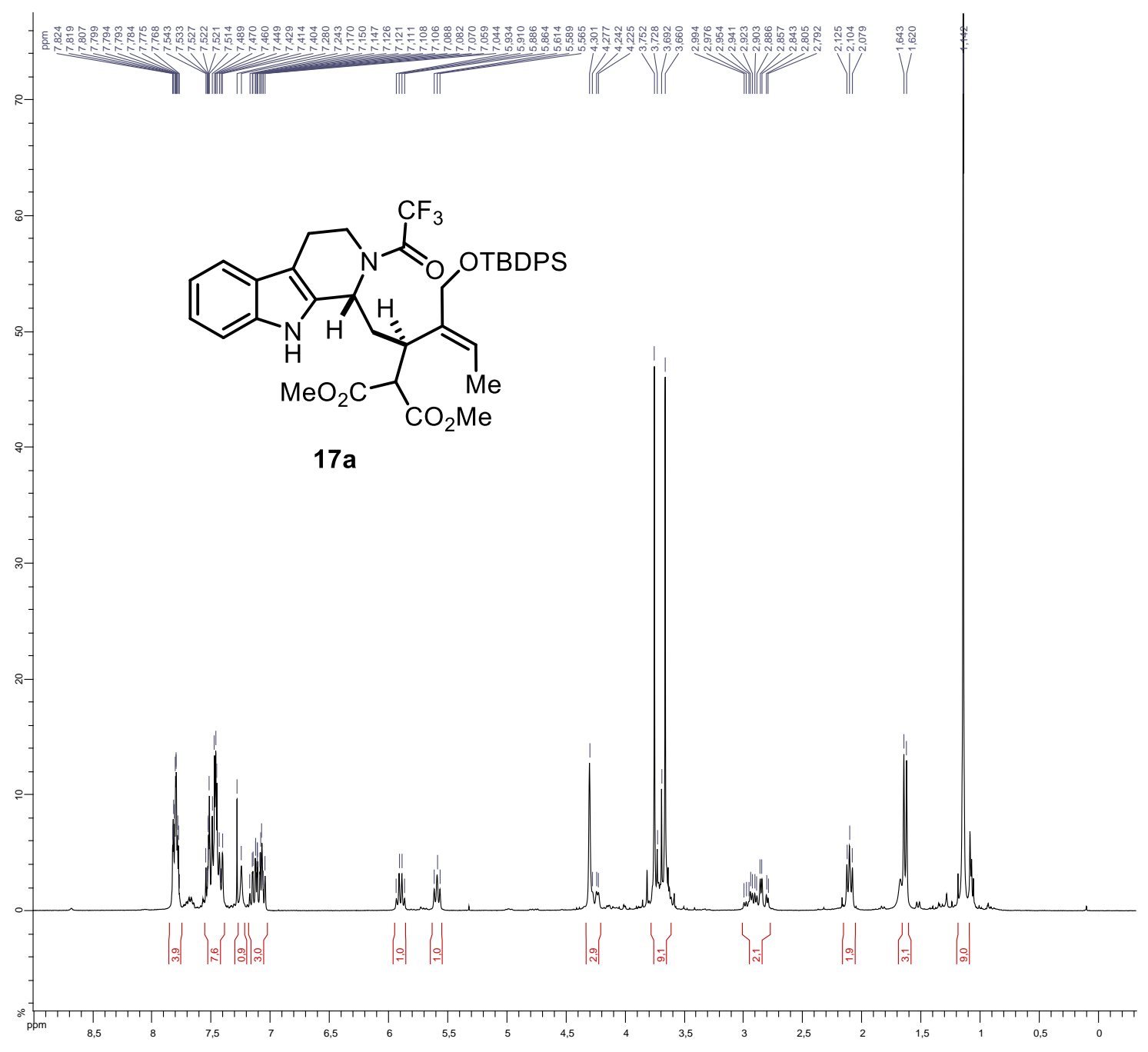


$13 \mathrm{C}\left\{{ }^{1} \mathrm{H}\right\} \mathrm{NMR}\left(\mathrm{CDCl}_{3}, 91 \mathrm{MHz}\right)$

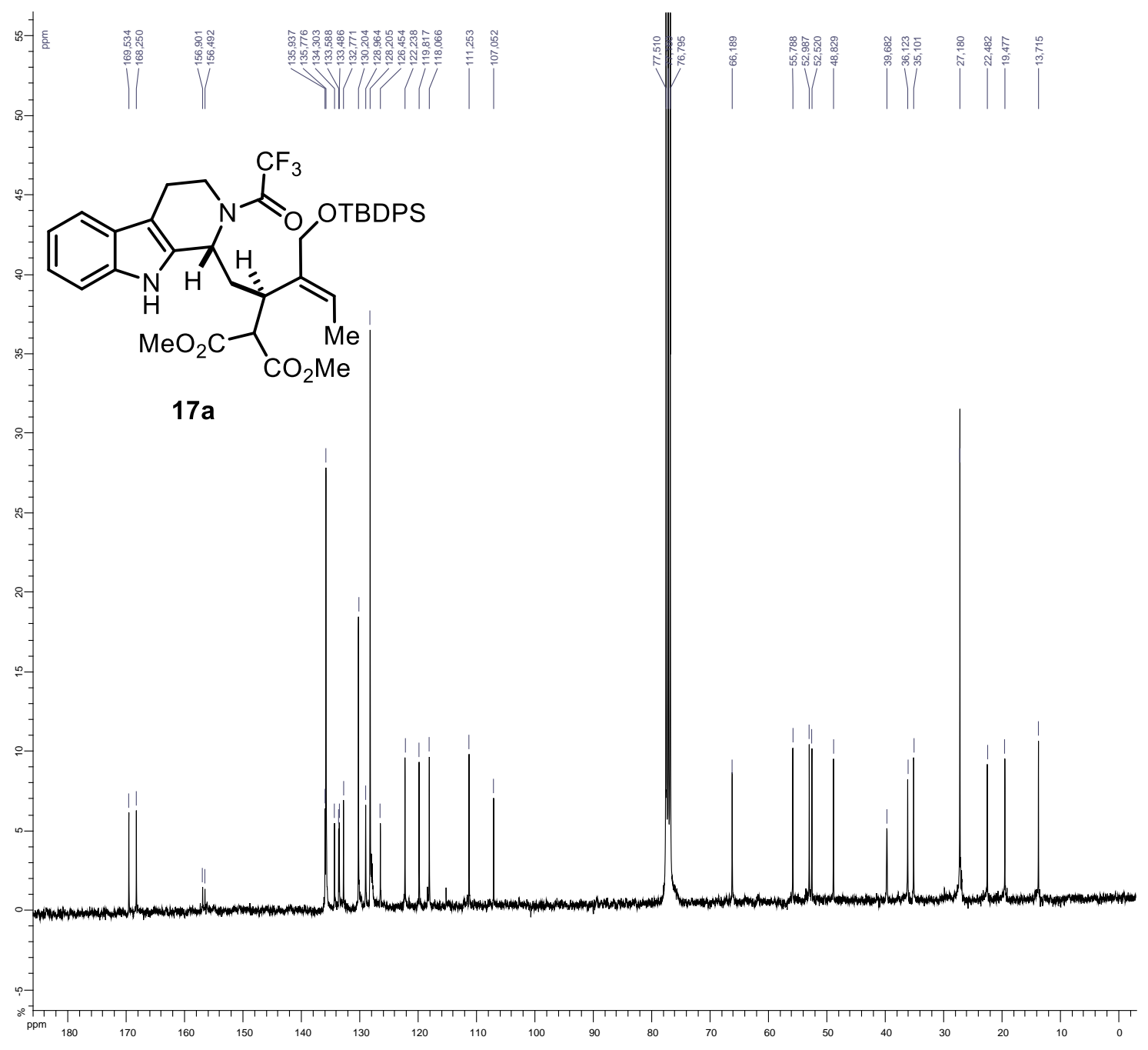


19F NMR $\left(\mathrm{CDCl}_{3}, 235 \mathrm{MHz}\right)$

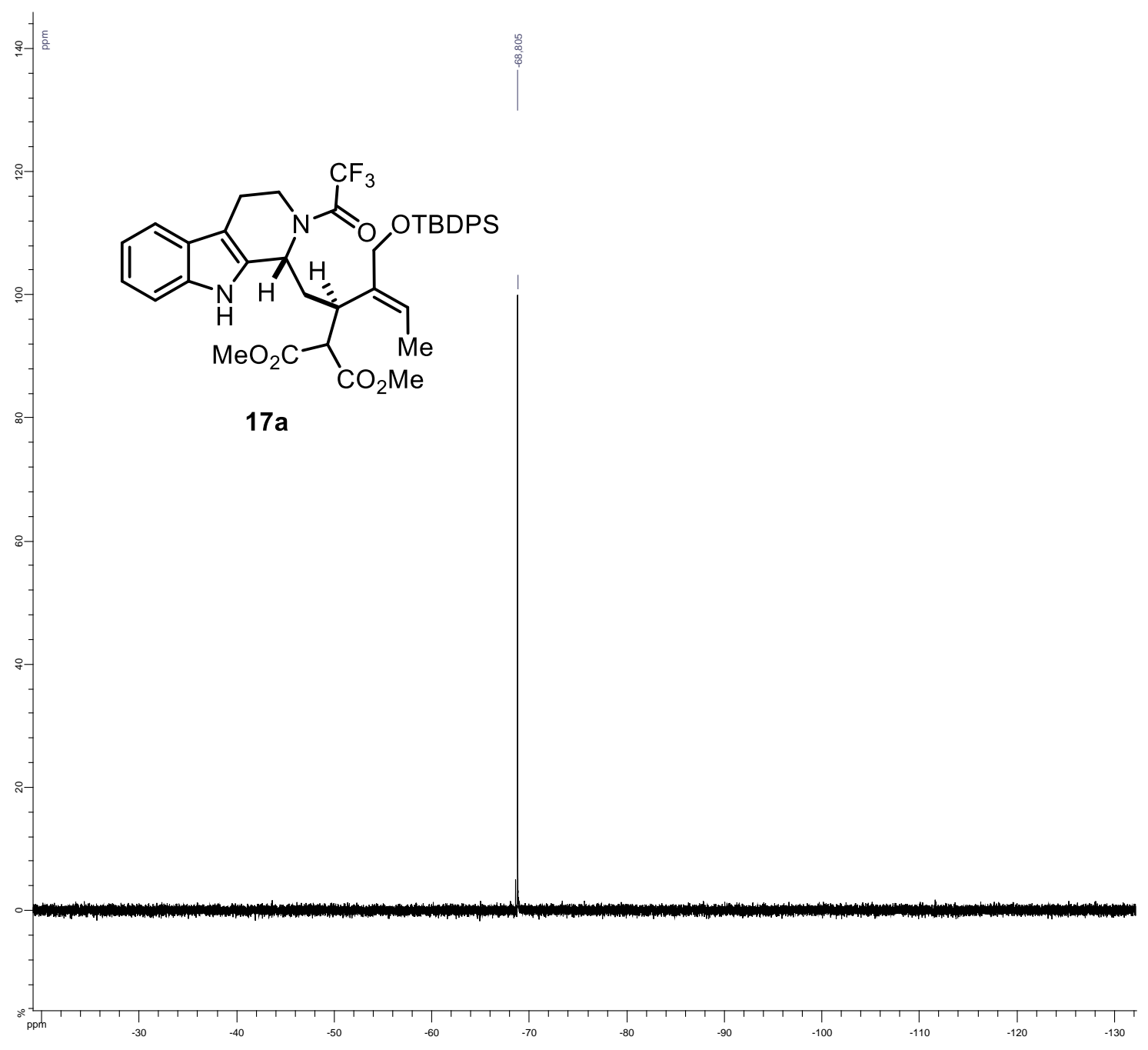


$1 \mathrm{H} \mathrm{NMR}\left(\mathrm{CDCl}_{3}, 300 \mathrm{MHz}\right)$




13C $\left\{{ }^{1} \mathrm{H}\right\} \mathrm{NMR}\left(\mathrm{CDCl}_{3}, 75 \mathrm{MHz}\right)$




19F NMR $\left(\mathrm{CDCl}_{3}, 235 \mathrm{MHz}\right)$

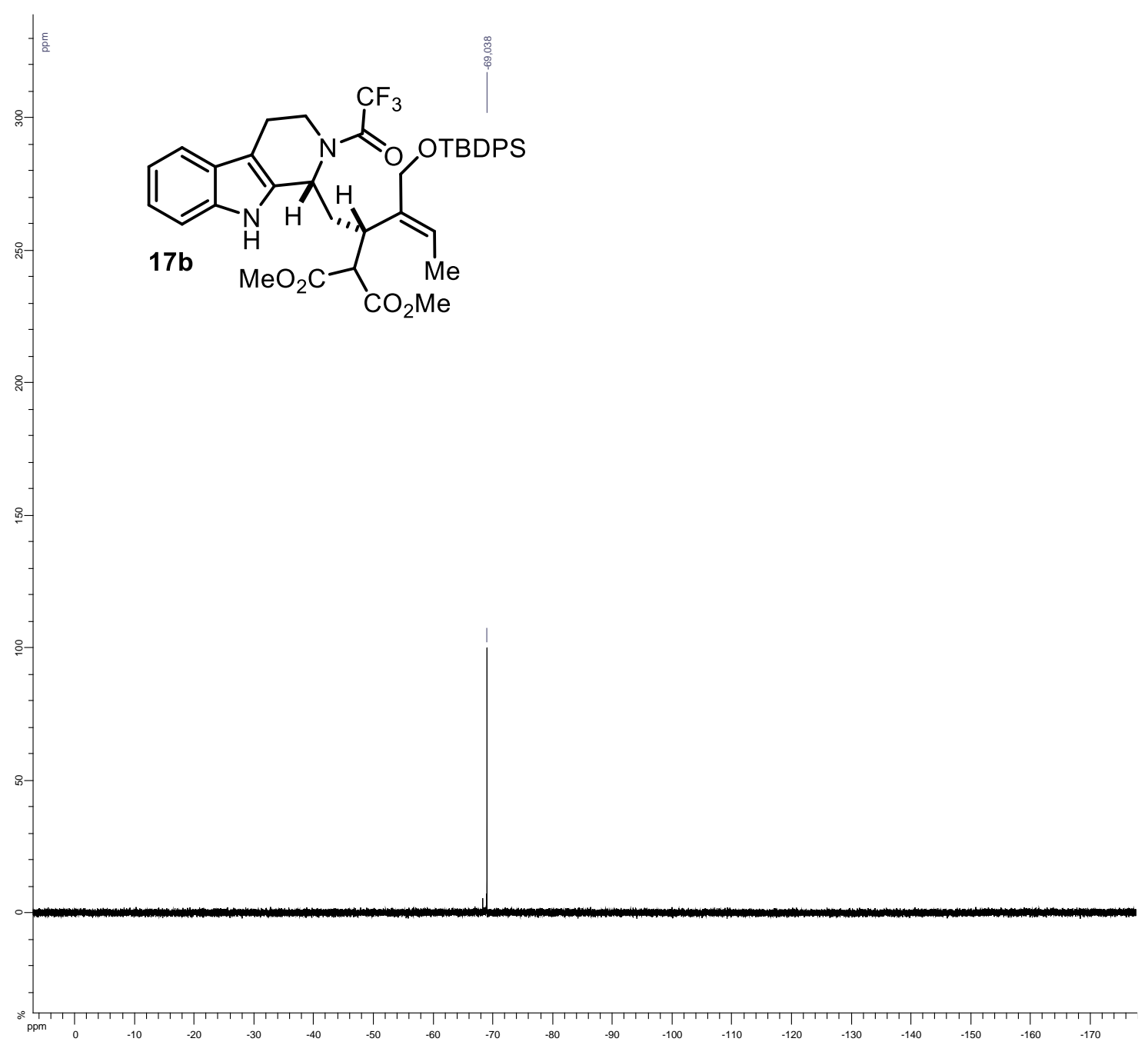


1H NMR $\left(\mathrm{CDCl}_{3}, 300 \mathrm{MHz}\right)$

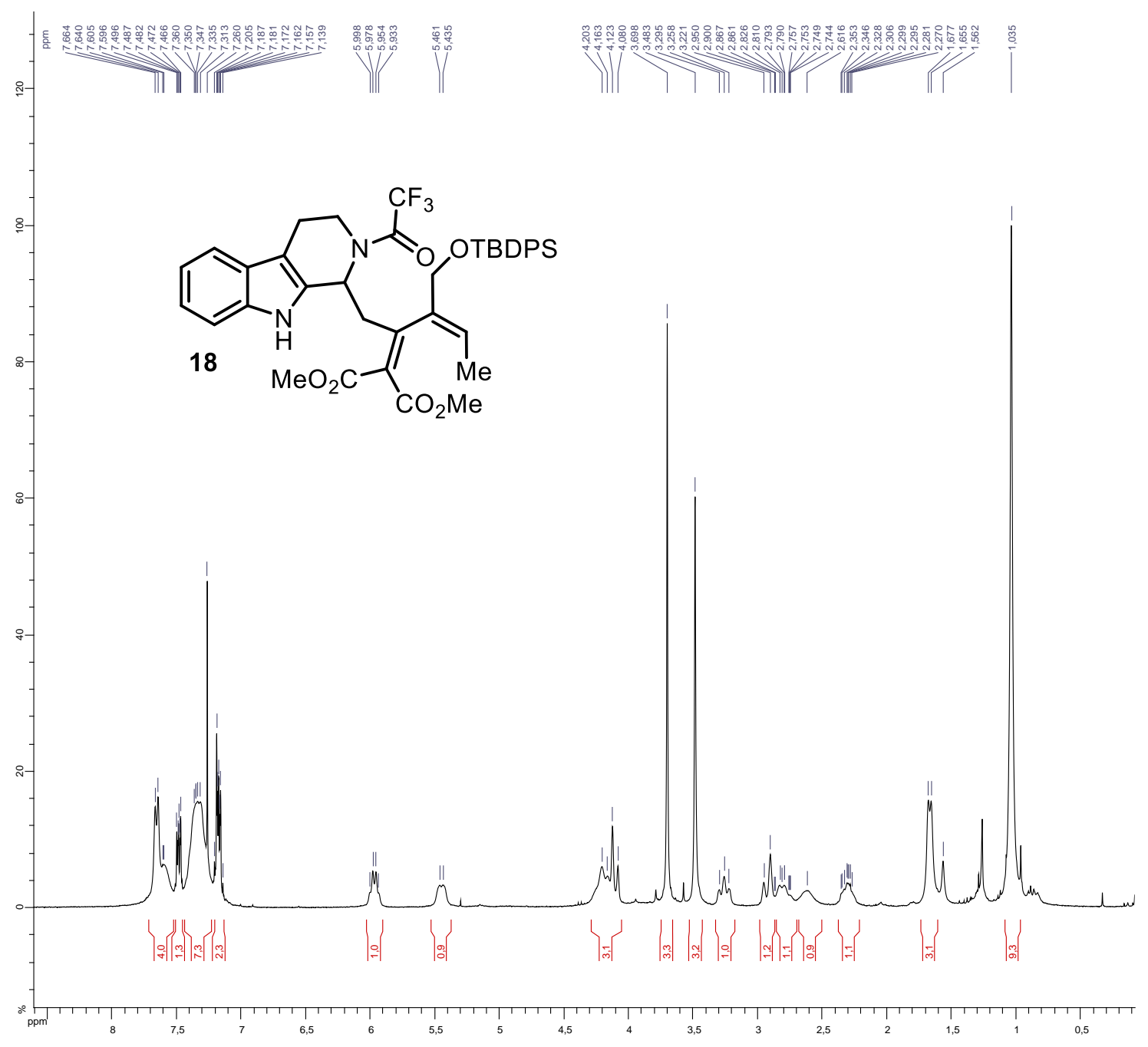


$13 \mathrm{C}\left\{{ }^{1} \mathrm{H}\right\} \mathrm{NMR}\left(\mathrm{CDCl}_{3}, 91 \mathrm{MHz}\right)$

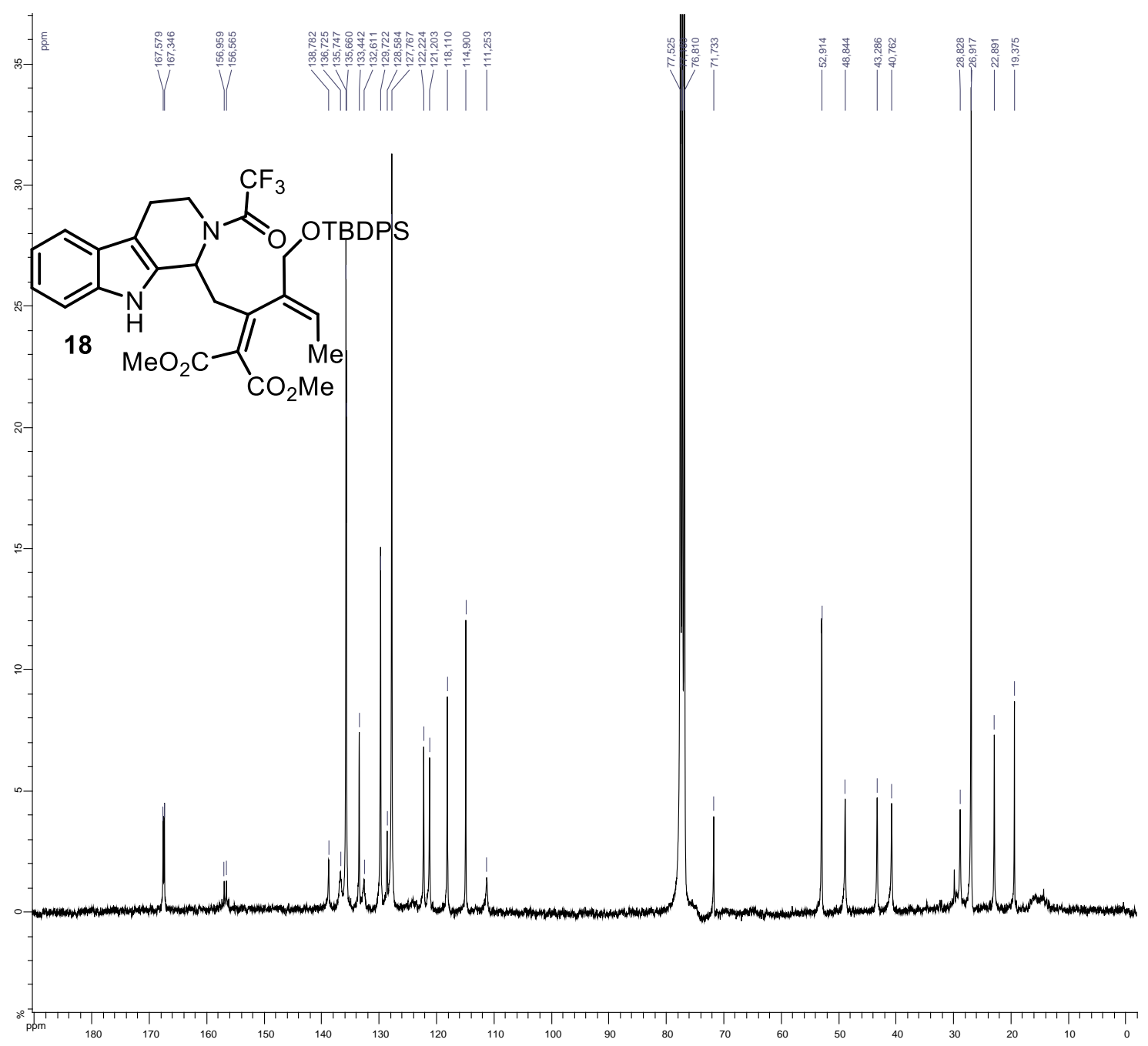


19F NMR $\left(\mathrm{CDCl}_{3}, 235 \mathrm{MHz}\right)$

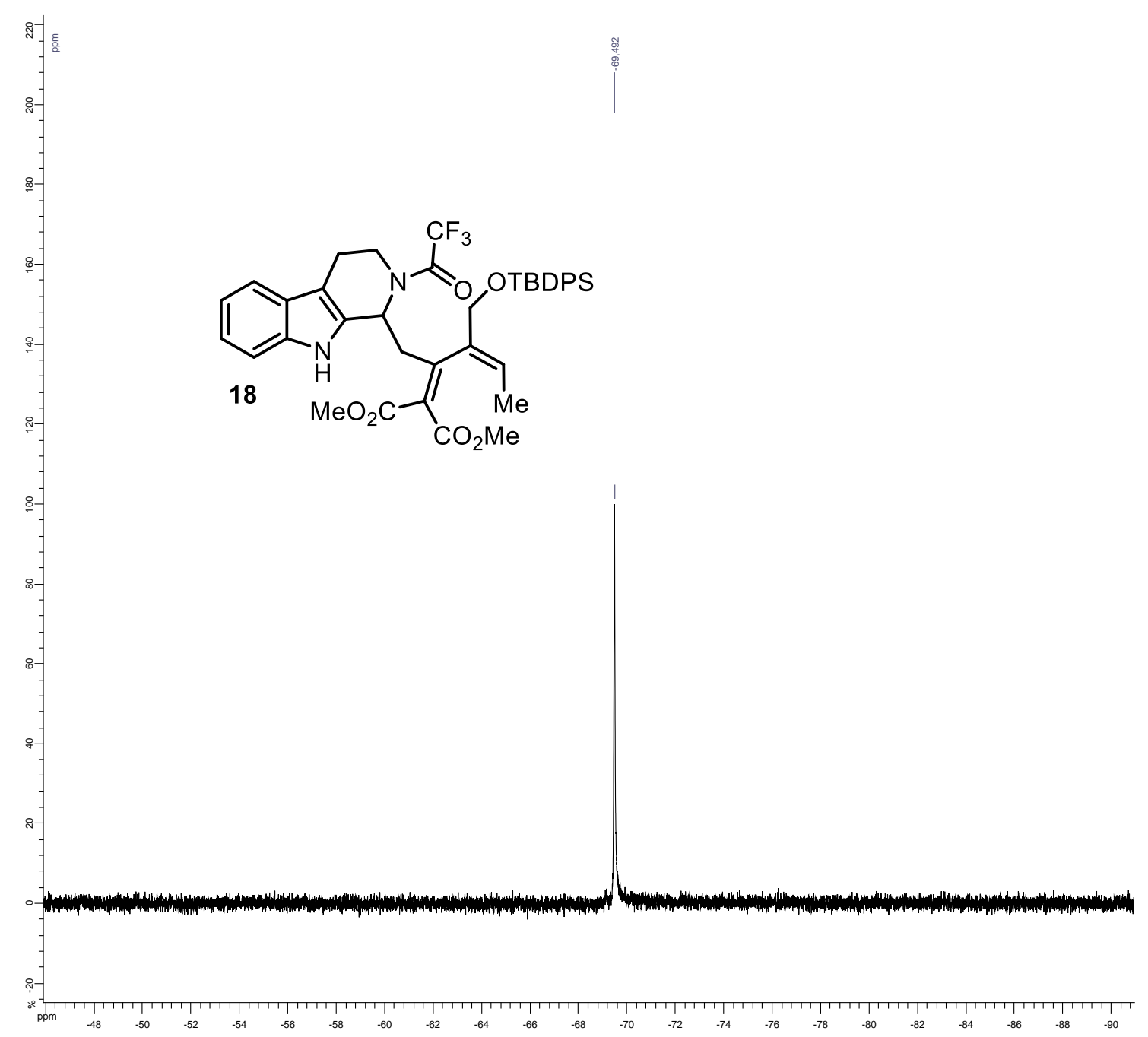




\section{X-ray analysis $17 a$}



Figure S1. ORTEP drawing for crystal structure of compound 17a.

X-ray diffraction data was collected by using a Kappa X8 APPEX II Bruker diffractometer with graphite-monochromated MoK $\alpha$ radiation $(\lambda=0.71073 \AA$ ). Crystal was mounted on a CryoLoop (Hampton Research) with Paratone-N (Hampton Research) as cryoprotectant and then flashfrozen in a nitrogen-gas stream at $100 \mathrm{~K}$. The temperature of the crystal was maintained at the selected value by means of a 700 series Cryostream cooling device to within an accuracy of $\pm 1 \mathrm{~K}$. The data were corrected for Lorentz polarization, and absorption effects. The structures were solved by direct methods using SHELXS-97 and refined against $F^{2}$ by full-matrix leastsquares techniques using SHELXL-2018 ${ }^{\mathrm{ii}}$ with anisotropic displacement parameters for all nonhydrogen atoms. Hydrogen atoms were located on a difference Fourier map and introduced into the calculations as a riding model with isotropic thermal parameters. All calculations were performed by using the Crystal Structure crystallographic software package WINGX.iii

The crystal data collection and refinement parameters are given in Table S 1 .

CCDC 2078456 contains the supplementary crystallographic data for this paper. These data can be obtained free of charge from the Cambridge Crystallographic Data Centre via http://www.ccdc.cam.ac.uk/Community/Requestastructure. 
Table S1. Crystallographic data and structure refinement details for compounds.

\begin{tabular}{|c|c|}
\hline Compound & $17 \mathbf{a}$ \\
\hline Empirical Formula & $\mathrm{C}_{40} \mathrm{H}_{45} \mathrm{~F}_{3} \mathrm{~N}_{2} \mathrm{O}_{6} \mathrm{Si}$ \\
\hline$M_{r}$ & 734.87 \\
\hline Crystal size, $\mathrm{mm}^{3}$ & $0.24 \times 0.19 \times 0.03$ \\
\hline Crystal system & monoclinic \\
\hline Space group & $P 21 / n$ \\
\hline a, $\AA$ & $12.6013(4)$ \\
\hline $\mathrm{b}, \AA$ & $23.0803(7)$ \\
\hline $\mathrm{c}, \AA$ & $13.2024(4)$ \\
\hline$\alpha,{ }^{\circ}$ & 90 \\
\hline$\beta,^{\circ}$ & $106.891(2)$ \\
\hline$\gamma,{ }^{\circ}$ & 90 \\
\hline Cell volume, $\AA^{3}$ & $3674.2(2)$ \\
\hline $\mathrm{Z} ; \mathrm{Z}^{\prime}$ & $4 ; 1$ \\
\hline $\mathrm{T}, \mathrm{K}$ & $100(1)$ \\
\hline $\mathrm{F}_{000}$ & 1552 \\
\hline$\mu, \mathrm{mm}^{-1}$ & 0.129 \\
\hline$\theta$ range, ${ }^{\circ}$ & $1.765-30.600$ \\
\hline Reflection collected & 114121 \\
\hline Reflections unique & 11283 \\
\hline $\mathrm{R}_{\mathrm{int}}$ & 0.1056 \\
\hline GOF & 1.008 \\
\hline Refl. obs. $(I>2 \sigma(I))$ & 7259 \\
\hline Parameters ; restraints & $475 ; 0$ \\
\hline $\mathrm{wR}_{2}$ (all data) & 0.1125 \\
\hline $\mathrm{R}$ value $(I>2 \sigma(I))$ & 0.0478 \\
\hline Largest diff. peak and hole $\left(\mathrm{e}-. \AA^{-3}\right)$ & $0.384 ;-0.339$ \\
\hline
\end{tabular}

\section{References}

1) Sheldrick, G. M. SHELXS-97, Program for Crystal Structure Solution, University of Göttingen, Göttingen, Germany, 1997.

2) Sheldrick, G. M. SHELXL-97, Program for the refinement of crystal structures from diffraction data, University of Göttingen, Göttingen, Germany, 1997.

3) Farrugia, L. J. J. Appl. Cryst., 1999, 32, 837. 